\title{
SYLOW $p$-SUBGROUPS OF THE GENERAL LINEAR GROUP OVER FINITE FIELDS OF CHARACTERISTIC $p$
}

\author{
A. J. WEIR
}

If $K$ is the finite field $G F(q)$ with $q=p^{k}$ elements then the general linear group $G L_{n}(K)$ has order

$$
q^{n(n-1) / 2}(q-1) \cdots\left(q^{n}-1\right) .^{1}
$$

Let $e_{i j}$ denote the matrix with the 1 of $K$ in the $(i, j)$ position and 0 elsewhere; we shall call any matrix of the form $1+\sum_{i<j} a_{i j} e_{i j}$ 1-triangular. The group $G_{n}$ of all 1-triangular matrices in $G L_{n}(K)$ is a Sylow $p$-subgroup of $G L_{n}(K)$. We shall often write $G$ for $G_{n}$ if this is unambiguous. $p$ is assumed throughout to be an odd prime.

The generators $1+a e_{i, i+1}$ and the fundamental relations connecting them are studied carefully in a recent paper by Pavlov ${ }^{2}$ (for the particular case $q=p$ ) and we have therefore mentioned them briefly in the opening paragraph.

When $i<j$ the group $P_{i j}$ of all $1+a e_{i j}(a \in K)$ is isomorphic to the additive group of $K$. Any subgroup $P$ of $G$ generated by these $P_{i j}$ is characterised by a partition diagram $|P|$. These partition diagrams bear a strong resemblance to the row of "hauteurs" which define the "sous-groupes parallélotopiques" of the Sylow $p$-subgroups of the symmetric groups on $p^{n}$ symbols, studied by Kaloujnine. ${ }^{3}$ A necessary and sufficient condition is given for the partition subgroup $P$ to be normal in $G$ and if $P^{\prime}=(P, G), P^{*} / P=$ centre of $G / P$, the duality between $P^{\prime}$ and $P^{*}$ is emphasised by constructing their partition diagrams.

Certain "diagonal" automorphisms are introduced and used to prove that any characteristic subgroup of $G$ is a normal partition subgroup. The maximal abelian normal subgroups are fully investigated and used in conjunction with the symmetry about the second diagonal to give a simple combinatorial proof that the characteristic subgroups of $G$ are precisely those given by symmetric normal partitions. In the last section we finally identify the group of automorphisms of $G$.

Received by the editors February 15, 1954.

1 Dickson, Linear groups.

2 P. P. Pavlov, Sylow p-subgroups of the full linear group over a simple field of characteristic p, Izvestiya Akad. Nauk SSSR, Ser. Mat. vol. 16 (1952) pp. 437-458. [Russian]. Math. Reviews vol. 14 (1953) p. 533.

${ }^{8} \mathrm{~L}$. Kaloujnine, La structure des p-groupes de Sylow des groupes symétriques finis, Ann. Ecole Norm. vol. 65 (1948) pp. 239-276. 
This paper is substantially the content of Chapter 5 of my Cambridge University Ph.D. Thesis (1953) and I should like to acknowledge here my gratitude to Professor Philip Hall who supervised this research in such a kind and encouraging way.

1. Generators of $G_{n}$.

$$
e_{i j} e_{h k}= \begin{cases}e_{i k} & \text { if } j=h, \\ 0 & \text { if } j \neq h .\end{cases}
$$

If $A=1+\sum_{i<j} a_{i j} e_{i j}$, then $r_{8}=\prod_{>>s}\left(1+a_{s j} e_{s j}\right)=1+\sum_{j>s} a_{s j} e_{s j}$ has the same sth row as $A$. Then $r_{n-1} r_{n-2} \cdots r_{1}=A$. Thus the set of all $1+a e_{i j}(a \in K, i<j)$ generate $G$.

Further if $u<v<w, a, b \in K$, we have the fundamental commutator relation

$$
\left(1+a e_{u v}, 1+b e_{v w}\right)=1+a b e_{u w} .
$$

Putting $b=1 ; u=i, v=i+1$ and $w=i+2, i+3, \cdots$ in succession we see that the set of elements $1+a e_{i, i+1}(a \in K ; i=1, \cdots, n-1)$ generate the group $G_{n}$.

2. The lower central series of $G_{n}$. We define $H_{k}$ to be the set of all $A$ for which $a_{i j}=0$ for $0<j-i<k$. If we write $\theta_{0}>\theta_{1}>\cdots$ for the derived series of $G$ we have the following

THEOREM 1. (i) The lower central series of $G_{n}$ coincides with the series $H_{1}>H_{2}>\cdots>H_{n}=1$.

(ii) $\left(H_{k}, H_{m}\right)=H_{k+m}$.

(iii) $\theta_{k}=H_{2}$.

Proof. Let $V_{k}$ be the set of all $L$ for which $1+L \in H_{k}$. We verify immediately that $V_{k} V_{m} \subset V_{k+m}$. It follows that $H_{k}$ is a group. Moreover if $1+L \in G$, then $1-L+L^{2} \cdots$ terminates and must therefore be $(1+L)^{-1}$.

Say $A=1+L \in H_{k}$ and $B=1+M \in H_{m}$ then

$$
\begin{aligned}
(A, B) & =(1+L)^{-1}\left\{(1+M)^{-1}+L-M L+O_{2 m+k}\right\}(1+M) \\
& =(1+L)^{-1}\left\{1+L+L M-M L+O_{2 m+k}\right\} \\
& =1+L M-M L+O_{2 m+k}+O_{2 k+m} \\
& =1+O_{k+m}, \quad \text { where } O_{k} \text { denotes "some element of } V_{k \cdot} \text { " }
\end{aligned}
$$

In other word $\left(H_{k}, H_{m}\right) \subset H_{k+m}$.

$H_{k}$ is generated (with some generators to spare, in general) by the set of all $1+a_{i j} e_{i j}\left(a_{i j} \in K, j-i \geqq k\right)$. If now $w-u \geqq k+m$ we may find $v$ so that $v-u \geqq k$ and $w-v \geqq m$, and we obtain the generators of 
$H_{k+m}$ in the form $1+a e_{u w}=\left(1+a e_{u v}, 1+e_{v w}\right)$. Hence $\left(H_{k}, H_{m}\right) \supset H_{k+m}$, and so finally $\left(H_{k}, H_{m}\right)=H_{k+m}$.

In particular $\left(H_{m}, H_{1}\right)=H_{m+1}$. Since $H_{1}=G, H_{1}>H_{2}>\cdots>H_{n}$ $=1$ is the lower central series of $G_{n}$.

The third part of the theorem follows immediately from the second by induction.

3. The partition subgroups. If $i<j$ the group $P_{i j}$ of all $1+a e_{i j}$ $(a \in K)$ is isomorphic to the additive group of $K$ and so is elementary abelian of order $q$. Any subgroup $P$ of $G$ generated by a selection of these $P_{i j}$ is called a partition subgroup. Such a subgroup may be characterised by a "partition" diagram $|P|$ in the natural way. For example (if $n \geqq 4$ ) the group generated by $P_{12}$ and $P_{24}$ contains also the subgroup $P_{14}$ and $|P|$ consists of the squares $(1,2),(2,4),(1,4)$. The sequence of diagrams for the lower central series is obtained from the whole diagram (representing $G$ ) by removing successive diagonals $j-i=1,2, \cdots$.

ThEOREM 2. A necessary and sufficient condition for the partition subgroup $P$ to be normal in $G$ is that the boundary of $|P|$ should move monotonically downward and to the right.

Proof. If $N$ is the least normal subgroup containing $1+a e_{i j}$, by the identity (1) it is clear that $N$ must also contain $P_{u j}$ and $P_{i v}$ where $u<i$ and $v>j$. Further since $P_{i v} \subset N$ we have $P_{u v} \subset N$ where $u<i$, $v>j$. If $\left|N_{i j}\right|$ consists of the squares $(u, v)$ with $u \leqq i, v \geqq j$ and if $\left|N_{i j}^{\prime}\right|$ is $\left|N_{i j}\right|$ omitting $(i, j)$, then $N$ must contain $N_{i j}^{\prime}$. The least normal subgroup containing $P_{i j}$ is $N_{i j}$. We shall find it convenient to refer to this process as "completing the rectangle." Now if $P$ is any normal partition subgroup and if $(i, j)$ is any square in $|P|$, then $P$ must contain $N_{i j}$. Conversely, the product of several $N_{i j}$ is a normal subgroup of $G$. These remarks are equivalent to the statement of the theorem.

Given two distinct squares $(i, j),(u, v)$ in $|G|$; if $u \leqq i, v \geqq j$ we shall say $(i, j)$ covers $(u, v)$. When $|P|$ is a normal partition we shall say $|P|$ covers $(u, v)$ if some square of $|P|$ covers $(u, v)$. If $(u, v)$ covers some square outside $|P|$ we shall say $(u, v)$ avoids $|P|$.

When $P$ is a normal partition subgroup we may define the groups $P^{\prime}=(P, G)$ and $P^{*}$ where $P^{*} / P=$ centre of $G / P$. Then $P^{\prime}$ and $P^{*}$ are again normal partition subgroups. More precisely

THEOREM 3. $\left|P^{\prime}\right|$ consists of the squares covered by $|P|$, and $\left|P^{*}\right|$ consists of the squares which do not avoid $|P|$.

Proof. Let $|N|$ be the set of squares covered by $|P|$. By the proc- 
ess of completing the rectangle we see that $P^{\prime}$ contains $N$.

Now $N$ is the product of normal subgroups $N_{i j}^{\prime}$ and so is normal. If $(i, j) \in|P|$, then $\left(1+a e_{i j}, 1+b e_{k m}\right) \in N$. Any commutator $(z, t)$ where $z \in P, t \in G$ may be expanded by application of the rule $(x y, r s)$ $=(x, s)^{y}(x, r)^{s y}(y, r)^{s \nu}(y, s){ }^{4}$ Hence $(P, G) \subset N$.

Let $|\bar{P}|$ be the set of squares which do not avoid $|P|$. We obtain $|\bar{P}|$ by adding one square to each row of $|P|$ except when this new square covers a square outside $|P|$. Clearly $(\bar{P}, G) \subset P$. If $A=1$ $+\sum_{i<j} a_{i j} e_{i j} \notin \bar{P}$ then $a_{i j} \neq 0$ for some $(i, j)$ avoiding $|P|$ and $(A, G)$ $\Phi P$. Hence $\bar{P}=P^{*}$. [We notice that the notation $N_{i j}^{\prime}$ already used is consistent with that of Theorem 3.]

Theorem 3 shows how strong is the duality between the groups $P^{\prime}$ and $P^{*}$. In particular we have as an immediate corollary

TheOREM 4. The upper and lower central series of $G$ coincide.

4. The diagonal automorphisms. If $W$ is the diagonal matrix

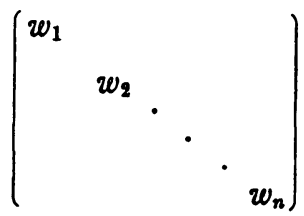

in $G L_{n}(K)$ and $A=1+\sum_{i<j} a_{i j} e_{i j} \in G_{n}$, then $W^{-1} A W=1+\sum_{i<j} a_{i j}^{*} e_{i j}$ where $a_{i j}^{*}=w_{i}^{-1} a_{i} w_{j}$. Let $D$ be the group of all such $W$.

Proposition. $D G_{n}$ is the normalizer of $G_{n}$ in $G L_{n}(K)$.

Proof. Clearly $D G_{n}$ is contained in this normalizer.

Suppose $M=\sum_{i, j} b_{i j} e_{i j}$ where $b_{u v} \neq 0(u>v)$, and $v$ is as small as possible with respect to this property.

On the one hand $\left(1+e_{v u}\right) M=M+\sum_{j} b_{u j} e_{v j}$ and this differs from $M$ in the $(v, v)$ position. On the other hand $M\left(1+\sum_{r<s} a_{r s} e_{r s}\right)$ has in the $(v, v)$ position the element $b_{v v}+\sum b_{v r} \sum_{r<v} a_{r v}=b_{v v}$ since the choice of $b_{u v}$ implies that $b_{v r}=0$ for all $r<v$. Now $1+e_{v u} \in G_{n}$ and we have shown that $M^{-1}\left(1+e_{v u}\right) M \notin G_{n}$. Thus $M$ does not belong to the normalizer of $G_{n}$ in $G L_{n}(K)$.

Any automorphism of $G$ of the form $A \rightarrow W^{-1} A W$ where $W \in D$ is called a diagonal automorphism. Let $\mathcal{D}$ be the group of all diagonal automorphisms.

5. The normal partition subgroups. It is now possible to prove the following

4. Hall, A contribution to the theory of groups of prime-power order, Proc. London Math. Soc. vol. 36 (1933). 
TheOREM 5. Any subgroup of $G$ which is invariant under the inner and diagonal automorphisms is a normal partition subgroup.

Proof. Any matrix of $G_{n+1}$ is expressible in the form

$$
\left(\begin{array}{ll}
1 & a \\
0 & A
\end{array}\right)=\left(\begin{array}{ll}
1 & 0 \\
0 & A
\end{array}\right)\left(\begin{array}{ll}
1 & a \\
0 & 1
\end{array}\right)=U V, \text { say }
$$

where $A \in G_{n}$ and $a$ is a row with elements in $K$.

The group of all $V$ is elementary abelian of order $q^{n}$ and is normal in $G_{n+1}$. In this way it is possible to express $G_{n+1}$ as the split extension $G_{n+1} \cong G_{n} H\left(G_{n} \cap H=1\right)$.

The theorem is true for $G_{2}$ and we assume it to be true for $G_{n}$. Suppose $R$ is a subgroup of $G_{n+1}$ which is invariant under the inner and diagonal automorphisms of $G_{n+1}$. Then $R \cap G_{n}$ is a subgroup of $G_{n}$ which is invariant under the inner and diagonal automorphisms of $G_{n}$ and so by the induction hypothesis is a normal partition subgroup of $G_{n}$.

$R \cap H$ is a subgroup of $H$ which is normal in $G_{n+1}$ and invariant under diagonal automorphisms. Hence $H$ is of the form $N_{1 j}$. \{If $a=\left(\alpha_{2}, \cdots, \alpha_{n+1}\right)$ and $\alpha_{j} \neq 0$ then $H$ contains $\left.P_{1, n+1}, P_{1 n}, \cdots, P_{1 j}.\right\}$

It is now sufficient to show that $R=\left(R \cap G_{n}\right)(R \cap H)$ for then the theorem follows by induction.

Clearly $R \supset\left(R \cap G_{n}\right)(R \cap H)$. The most general element of $R$ is of the form

$$
\left(\begin{array}{ll}
1 & 0 \\
0 & A
\end{array}\right)\left(\begin{array}{ll}
1 & a \\
0 & 1
\end{array}\right)=U V, \text { say. }
$$

If

$$
W=\left(\begin{array}{ll}
1 & 0 \\
0 & 2
\end{array}\right)
$$

then

$$
W^{-1} U V W=U V^{2} \in R \quad(p \neq 2) .
$$

Hence $U, V \in R$. In other words $R \subset\left(R \cap G_{n}\right)(R \cap H)$.

REMARK. Since the diagonal automorphisms clearly leave invariant any partition subgroup, the converse of Theorem 5 is also true and so we may characterise the normal partition subgroups as those which are left invariant by the inner and diagonal automorphisms.

There is a further important automorphism of $G_{n}$ which we may regard as a symmetry about the second diagonal: 
$\tau: 1+\sum a_{i j} e_{i j} \rightarrow 1+\sum b_{i j} e_{i j}$ where $b_{i j}=a_{n+1-j, n+1-i}$.

In view of this we have the important

COROLlaRY. Every characteristic subgroup of $G$ is a "symmetric" normal partition subgroup.

6. The maximal abelian normal subgroups. The derived group $\theta_{1}$ of $G$ gives the maximal abelian quotient group. A natural dual of $\theta_{1}$ would be a maximal abelian normal subgroup. ${ }^{5}$ We shall determine the set of all maximal abelian normal subgroups of $G$.

If $A_{i}=N_{i, i+1}(i=1,2, \cdots, n-1)$ then $A_{i}$ is clearly a normal (partition) subgroup. If $(u, v)$ and $\left(u^{\prime}, v^{\prime}\right) \in\left|A_{i}\right|$ then $u^{\prime} \leqq i<v$ and $\left(P_{u v}, P_{u^{\prime} v^{\prime}}\right)=1$. Hence $A_{i}$ is abelian. If $z \notin A_{i}$, that is if

$$
z=1+\sum_{u<v} a_{u v} e_{u v}
$$

and some $a_{u v} \neq 0$ where $u>i$ or $v \leqq i$, then $G p\left[z, A_{i}\right]$ is not abelian.

(i) Say $u>i$. $\left(1-e_{i u}\right) z\left(1+e_{i u}\right)$ differs from $z$ in $(i, v)$ position.

(ii) Say $v \leqq i$. $\left(1-e_{v, i+1}\right) z\left(1+e_{v, i+1}\right)$ differs from $z$ in the $(u, i+1)$ position.

We have now shown that $A_{i}$ is a maximal abelian normal subgroup of $G_{n}(i=1,2, \cdots, n-1)$.

A necessary and sufficient condition for $x \in G$ to belong to a maximal abelian normal subgroup of $G$ is that $x$ should commute with all its conjugates in $G$.

Consider $G_{3}$ : if $x=1+e_{12}+e_{23}$ then $x$ commutes with its conjugates all of which have the form $x+a e_{13}$ and so $x$ is in a maximal abelian normal subgroup (clearly neither $A_{1}$ nor $A_{2}$ ). Though the $A_{i}$ are the only partition subgroups which are maximal abelian normal we must expect other types of maximal abelian normal subgroups in general.

Suppose

$$
\begin{aligned}
& 1+L=1+\sum_{u<v} a_{u v} e_{u v} \quad\left(a_{u v} \in K\right), \\
& 1+M=1+\sum_{u<v} b_{u v} e_{u v} \quad\left(b_{u v} \in K\right)
\end{aligned}
$$

then $(1+L)(1+M)=1+L+M+L M$ and so $1+L, 1+M$ commute if and only if $L, M$ commute.

Now

$$
\left(1-e_{i j}\right)(1+L)\left(1+e_{i j}\right)=1+L+L e_{i j}-e_{i j} L
$$

5 H. Zassenhaus, The theory of groups, Chelsea, 1949, Chap. IV, 3.4, p. 115. 
since $e_{i j} L e_{i j}=0$. Suppose $1+L$ belongs to a maximal abelian normal subgroup; then we require $L$ to commute with $L e_{i j}-e_{i j} L$, in other words we require $L e_{i j} L-e_{i j} L^{2}-L^{2} e_{i j}+L e_{i j} L=0$ or $2 L e_{i j} L=e_{i j} L^{2}$ $+L^{2} e_{i j}$. Now

$$
L e_{i j}=\sum_{u<i} a_{u i} e_{u j}, \quad e_{i j} L=\sum_{j<v} a_{j v} e_{i v} .
$$

Hence we require

$$
2 \sum_{j<t} \sum_{u<i} a_{u i} a_{j t} e_{u t}=\sum_{j<v<t} a_{j v} a_{v t} e_{i t}+\sum_{w<u<i} a_{w u} a_{u i} e_{w j} .
$$

Each of these three sums belongs to a separate part of the partition diagram of $G$, and so they all vanish $[p \neq 2]$.

We thus have the following three sets of equations:

$$
\begin{aligned}
& a_{1 i} \cdot a_{j, j+1}=a_{1 i} \cdot a_{j, j+2}=\cdots=a_{1 i} \cdot a_{j n}=0, \\
& a_{2 i} \cdot a_{j, j+1}=a_{2 i} \cdot a_{j, j+2}=\cdots=a_{2 i} \cdot a_{j n}=0 \text {, } \\
& a_{i-2, i} a_{j, j+1}=a_{i-2, i} a_{j, j+2}=\cdots=a_{i-2, i} a_{j n}=0 \text {, } \\
& a_{i-1, i} a_{j, j+1}=a_{i-1, i} a_{j, j+2}=\cdots=a_{i-1, i} a_{j n}=0 \text {, } \\
& a_{j, j+1} a_{j+1, j+2}=0 \text {, }
\end{aligned}
$$$$
a_{j, j+1} a_{j+1, j+3}+a_{j, j+2} a_{j+2, j+3}=0,
$$$$
a_{j, j+1} a_{j+1, n}+a_{j, j+2} a_{j+2, n}+\cdots+a_{j, n-1} a_{n-1, n}=0,
$$$$
a_{12} a_{2 i}+a_{13} a_{3 i}+\cdots+a_{1, i-1} a_{i-1, i}=0,
$$$$
a_{23} a_{3 i}+\cdots \quad+a_{2, i-1} a_{i-1, i}=0,
$$

$$
\begin{aligned}
a_{i-3, i-2} a_{i-2, i}+a_{i-3, i-1} a_{i-1, i} & =0, \\
a_{i-2, i-1} a_{i-1, i} & =0,
\end{aligned}
$$

If there is one element $a_{u v}$ in the diagonal $v-u=1$ which does not vanish, then by the last equation of (i) every other element in the same diagonal which is not adjacent to $(u, v)$ must vanish, also the first equation of (ii) or the last equation of (iii) show that the adjacent ones vanish. Hence if $1+\sum_{u<v} a_{u v} e_{u v}$ belongs to a maximal abelian normal subgroup and has one nonzero element in the diagonal $v-u=1$, then all the other elements in this diagonal vanish.

Suppose now that the $v$ th column is the first which is not composed entirely of zeros and $a_{u v}$ the last nonvanishing element of it. In other 
words $a_{u v} \neq 0$ and $a_{i j}=0$ if $j<v$ and also if $j=v, i>u$. If $v=n, 1+L$ $\in A_{n-1}$ so we assume $v<n$.

There are two cases to consider: (a) $u>1$, (b) $u=1$.

(a) We may take $i=v$ in (i) and this gives $a_{u v} a_{j, j+1}=a_{u v} a_{j, j+2}$ $=\cdots=0$ provided $j>v$. Thus $a_{r s}=0$ whenever $r>v$.

Take $j=u$ in (ii), then in the equation

$$
a_{u, u+1} a_{u+1, m}+\cdots+a_{u v} a_{v m}+\cdots+a_{u, m-1} a_{m-1, m}=0
$$

all the $a_{r s}$ for which $s<v$ vanish by our choice of $a_{u v}$, and all the $a_{r s}$ for which $r>v$ vanish by the result we have just proved. Thus $a_{v m}=0$ (all $m>v$ ).

Finally we have $a_{r s}=0$ whenever $r \geqq v$, and we now see that in this case $1+L \in A_{v-1}$.

(b) We may take $i=v$ in (i) and we find just as before that $a_{r s}=0$ whenever $r>v$.

The first equation of (iii) is

$$
a_{12} a_{2 i}+a_{13} a_{3 i}+\cdots+a_{1 v} a_{v i}+\cdots+a_{1, i-1} a_{i-1, i}=0 .
$$

Now $a_{12}=\cdots=a_{1, v-1}=0$ by our choice of $a_{u v}$, and $a_{r \varepsilon}=0$ whenever $r>v$ by above, so that only one term remains in the equation. Thus $a_{v i}=0(v<i<n)$.

Finally $a_{r s}=0$ whenever $r \geqq v$ except possibly $a_{v n}$. If $a_{v n}=0$, then just as before $1+L \in A_{v-1}$.

However in fact $a_{v n}$ need not be zero and each of its possible $q-1$ nonzero values gives us a new maximal abelian normal subgroup. Any normal subgroup containing for example $x=1+e_{1 v}+c e_{v n}$ must contain all the conjugates of $x$ in $G$. Now $x^{-1}-1-e_{1 v}-c e_{v n}+c e_{1 n}$ and $\left(1-a e_{v w}\right) x\left(1+a e_{v w}\right)=x+a e_{1 w}$. Hence any normal subgroup containing $x$ must contain all $1+a e_{1 w}$ for $w>v, a \in K$ and similarly must contain all $1+a e_{w n}$ for $w<v, a \in K$.

Suppose now that $y$ belongs to an abelian normal subgroup containing $x$, and say $y=1+\sum_{i<j} a_{i j} e_{i j}$. Then $y$ must commute with $x$ and also with $1+e_{1 w}($ all $w>v)$ and $1+e_{w n}$ (all $\left.w<v\right)$. This shows that $a_{r s}=0$ if $r \geqq v$, also if $s \leqq v$, except possibly $a_{1 v} \neq 0$ or $a_{v n} \neq 0$ but in this case $c a_{1 v}=a_{v n}$.

Thus $N_{v}(c)=G p\left[1+a e_{1 v}+c a e_{v n},(a \in K)\right] N_{v-1, v+1}$ is the unique maximal abelian normal subgroup containing $x$.

The results of this section may be summarised in

Theorem 6. The maximal abelian normal subgroups of $G_{n}$ fall into two distinct classes:

$$
A_{i}=N_{i, i+1} \quad(i=1,2, \cdots, n-1),
$$




$$
N_{v}(c), \quad \text { where } c \neq 0, c \in K,(v=2, \cdots,[n+1 / 2]) .
$$

7. The characteristic subgroups. We have the following fundamental

THEOREM 7. The characteristic subgroups of $G$ are precisely the normal partition subgroups whose partitions are symmetric about the second diagonal.

Proof. We consider the effect of an automorphism $\theta$ on the maximal abelian normal subgroups. Certainly it is clear that these must be permuted among themselves. All of the "exceptional" maximal abelian normal subgroups $N_{v}(c)$ except for $v=2$ are contained in $H_{2}$ and $\mathrm{H}_{2}$ is characteristic in $G$. Also no $A_{i}$ is contained in $\mathrm{H}_{2}$ so we expect the $A_{i}(1<i<n-1)$ to be permuted by $\theta$. These $A_{i}$ divide naturally into pairs of groups with the same order, and for example we see that $A_{2}$ transforms under $\theta$ into itself or into $A_{n-2}$. Moreover $\theta$ leaves $A_{2}$ invariant if and only if $\theta$ leaves $A_{n-2}$ invariant. Hence both $A_{2} A_{n-2}$ and $N_{2, n-1}=A_{2} \cap A_{n-2}$ are characteristic subgroups of $G_{n}$.

The join of $A_{1}, A_{n-1}$ and $N_{2}(c)$ is just $A_{1} A_{n-1}$ and this again is characteristic in $G_{n}$.

If we write $r^{\prime}=n+1-r$, then $\tau$ sends $P_{r s}$ into $P_{s^{\prime} r^{\prime}}$. Any symmetric normal partition subgroup may be built up as a join of $N_{r s} N_{s^{\prime} r^{\prime}}$ $(r=1,2, \cdots)$. But these may all be obtained as intersections of groups which we have shown to be characteristic. For example we intersect $A_{2} A_{n-2}$ successively with $A_{3} A_{n-3}, A_{4} A_{n-4}, \cdots$ and then the square partition subgroups $N_{r r^{\prime}}$ to obtain every $N_{28} N_{s^{\prime}, n-1}$. Combining these results with the corollary to Theorem 5 we have the above theorem.

8. The automorphisms of $G_{n}$. Since the automorphisms of $G$ have been completely determined by Palov $^{2}$ for the case of a ground field with $p$ elements, we shall sketch the parts of this section which are merely generalizations of his work, and we shall also try as far as possible to use his notation.

The group $\mathfrak{J}$ of inner automorphisms is isomorphic to $G_{n} / H_{n-1}$ and so has order $q^{\left(n^{2}-n-2\right) / 2}$.

The diagonal automorphism induced by the diagonal matrix $W$ is the identity if and only if $W$ is a scalar matrx. Hence $\mathcal{D}$ has order $(q-1)^{n-1}$.

The ground field $K$ may be regarded as a vector space of dimension $k$ over the field $G F(p)$ of integers $\bmod p$. Let $a_{1}, \cdots, a_{k}$ be a basis. The group $G L_{k}(p)$ of all nonsingular linear transformations of $K$ induces a group $\mathcal{L}$ of automorphisms of $G$ : if $g \in G L_{k}(p)$ then $\gamma$ is the 
induced automorphism which maps the generators $1+a_{i} e_{r, r+1}$ into $1+a_{i}^{0} e_{r, r+1}(i=1, \cdots, k ; r=1, \cdots, n-1)$.

$\mathcal{L} \cap D$ consists of the automorphisms induced by matrices of $D$ of the form

$$
\left(\begin{array}{lllll}
a & & & & \\
& a^{2} & & \\
& & \cdot & & \\
& & & \cdot \\
& & & a^{n}
\end{array}\right),
$$$$
a \neq 0, a \in K \text {. }
$$

For each $i=1, \cdots, k ; r=1, \cdots, n-1$ there is a central automorphism $\tau_{r}^{i}$ which maps the one generator $1+a_{i} e_{r, r+1}$ into $1+a_{i} e_{r, r+1}$ $+b_{i} e_{1 n}$ (where $b_{i}$ is an arbitrary element of $K$ ), and leaves the other generators invariant. For $r=1$ and $r=n-1$ these are already inner automorphisms. Let $Z$ be the group generated by $\tau_{r}^{i}(i=1, \cdots, k$; $r=2, \cdots, n-2)$, then $Z$ is elementary abelian of order $q^{k(n-3)}$.

There are two types of extremal automorphisms

$$
\sigma_{1}(b): 1+a e_{12} \rightarrow 1+a e_{12}+a b e_{2 n} \quad(b \in K),
$$

and

$$
\sigma_{2}(b): 1+a e_{n-1, n} \rightarrow 1+a e_{n-1, n}+a b e_{1, n-1} \quad(b \in K) .
$$

The group $V$ generated by the extremal automorphisms is elementary abelian of order $q^{2}$. We write $\mathcal{P}=Z \mathcal{Z}$. (This is a direct product.)

ThEOREM 8. The group $A$ of all automorphisms of $G$ is generated by the subgroups $[\tau], \mathcal{L}, \mathcal{D}, \mathfrak{J}, \mathcal{P}$.

Proof. If $\alpha$ is an automorphism which leaves $H_{2}$ elementwise invariant and which induces the identity automorphism on $G / H_{2}$, then $\alpha$ may be obtained by multiplying each element of $G_{n}$ by an element in the centre $\left(H_{n-2}\right)$ of $H_{2} \cdot{ }^{6}$ The central automorphisms are clearly of this type.

If $\left(1+e_{r, r+1}\right)^{\alpha}=1+e_{r, r+1}+b e_{2 n}$ and $r>1$, by commuting with $1+e_{12}$ we find an element in $\mathrm{H}_{2}$ which is not invariant unless $b=0$.

If $\left(1+e_{12}\right)^{\alpha}=1+e_{12}+b e_{2 n}$, then since $1+e_{12}, 1+a e_{12}$ commute we must have $\left(1+a e_{12}\right)^{\alpha}=1+a e_{12}+a b e_{2 n}$. There is a similar argument involving $1+a e_{n-1, n}$. It is now clear that $\alpha \in \mathcal{P}$.

It remains to be shown that if $\alpha$ is any automorphism of $G$ then we may (simultaneously) copy the effect of $\alpha$ on $H_{2}$ and on $G / H_{2}$ using only the automorphisms of $[\tau], \mathcal{L}, \mathcal{D}$ and $\mathfrak{J}$.

Under an automorphism $\alpha$ the subgroups $A_{i}$ are either all left in-

' H. Zassenhaus, The theory of groups, Chap. 2, Exercise 6, p. 78. 
variant or are all reflected in the second diagonal. By multiplying by $\tau$ if necessary we may assume that $\alpha$ leaves each $A_{i}$ invariant.

If $\left(1+a e_{12}\right)^{\alpha}=1+\bar{a} e_{12}+\cdots$ (where the extra terms are in $N_{13}$ ) then $\left\{1+(r a+s b) e_{12}\right\} \alpha=1+(r \bar{a}+s \bar{b}) e_{12}+\cdots$ where $r, s$ are integers $\bmod p$. Hence $\alpha$ induces a linear transformation $a \rightarrow \vec{a}$ of the vector space $K$.

The set $\left\{1+a_{i} e_{r, r+1} ;(i=1, \cdots, k ; r=1, \cdots, n-1)\right\}$ is a minimal system of generators of $G_{n}$. Hence $\left\{\left(1+a_{i} e_{12}\right)^{\alpha} ;(i=1, \cdots, k)\right\}$ is part of a minimal system of generators of $G_{n}$ and $\bar{a}_{1}, \cdots, \bar{a}_{k}$ is again a basis of the vector space $K$. The linear transformation $a \rightarrow \bar{a}$ is thus nonsingular.

If $\left(1+a e_{23}\right)^{\alpha}=1+a^{\prime} e_{23}+\cdots, a \rightarrow a^{\prime}$ is again a linear transformation of $K$. Now the commutator $\left(1+a e_{12}, 1+b e_{23}\right)=1+a b e_{13}$ has the same value if we interchange $a$ and $b$, and so $1+\bar{a} b^{\prime} e_{13}+\cdots$ $=1+a^{\prime} b e_{13}+\cdots$. If $b \neq 0$, since $N_{12}$ cannot map into $N_{13}$ and $N_{23}$ cannot map into $N_{23}^{\prime}$ neither $b$ nor $b^{\prime}$ vanishes and $\bar{a} / \bar{b}=a^{\prime} / b^{\prime}$. The effect of $\alpha$ on $P_{12}$ is thus the same as the effect on $P_{23}$ apart from a constant factor. Since we may use a diagonal automorphism to give the required constant factors in $P_{23}, P_{34}, \cdots, P_{n-1, n}$ there is an element $\beta$ of $\mathcal{L} \mathcal{D}$ which has the same effect as $\alpha$ on $G_{n} \bmod H_{2}$. Let us divide through by $\beta$ and assume that $\alpha$ induces the identity on $G / H_{2}$.

We now look for an inner automorphism which has the same effect as $\alpha$ on $H_{2}$.

If, under $\alpha, 1+e_{23} \rightarrow 1+e_{23}+f e_{13}+a e_{24}\left(\bmod H_{3}\right)$ then by commuting with $1+d e_{12}$ we see that $1+d e_{13} \rightarrow 1+d e_{13}+d a e_{14}\left(\bmod H_{4}\right)($ all $d \in K)$. We transform by $1+a e_{34}$. This transformation also sends $1+e_{46} \rightarrow 1$ $+e_{46}-a e_{36}\left(\bmod H_{4}\right)$ and $1+e_{47} \rightarrow 1+e_{47}-a e_{37}\left(\bmod H_{5}\right)$ but this is a necessary contribution since $1+e_{13}, 1+e_{46}$ commute and $1+e_{67} \rightarrow 1$ $+e_{67}\left(\bmod H_{2}\right)$.

If, under $\alpha, 1+e_{24} \rightarrow 1+e_{24}+b e_{26}+c e_{14}\left(\bmod H_{4}\right)$ we transform by $1+b e_{45}-c e_{12}$. This transformation also affects $1+e_{57}$ and $1+e_{58}$ but here again there is a necessary contribution.

By such inner automorphisms using elements in $P_{i j}, j-i=1$, we copy the effect of $\alpha$ on $P_{i j}(j-i=2) \bmod H_{4}$ and $P_{i j}(j-i=3) \bmod H_{5}$. Since $H_{2}$ is generated by the $P_{i j}$ for which $j-i=2,3$ we finally obtain an inner automorphism which has the same effect as $\alpha$ on $\mathrm{H}_{2}$ by transforming successively by elements in $P_{i j}, j-i=1,2,3, \cdots$. This completes the proof of Theorem 8 .

Cambridge University 